\title{
Ultrafast optical recording reveals distinct capsaicin-induced ion dynamics along single nociceptive neurite terminals in vitro
}

Robert H. Goldstein

Ben Katz

Shaya Lev

Alexander M. Binshtok 


\title{
Ultrafast optical recording reveals distinct capsaicin-induced ion dynamics along single nociceptive neurite terminals in vitro
}

\author{
Robert H. Goldstein, ${ }^{a, b}$ Ben Katz, ${ }^{a, b}$ Shaya Lev, ${ }^{a, b}$ and Alexander M. Binshtok ${ }^{a, b, *}$ \\ ${ }^{a}$ The Hebrew University, Institute for Medical Research Israel Canada, Faculty of Medicine, Department of Medical Neurobiology, Jerusalem, Israel \\ ${ }^{\mathrm{b}}$ The Hebrew University, The Edmond and Lily Safra Center for Brain Sciences, Jerusalem, Israel
}

\begin{abstract}
Pain signals are detected by terminals of nociceptive peripheral fibers situated among the keratinocytes and epithelial cells. Despite being key structures for pain-related stimuli detection and transmission, little is known about the functional organization of terminals. This is mainly due to their minute size, rendering them largely inaccessible by conventional experimental approaches. Here, we report the implementation of an ultrafast optical recording approach for studying cultured neurite terminals, which are readily accessible for assay manipulations. Using this approach, we were able to study capsaicin-induced calcium and sodium dynamics in the nociceptive processes, at a near-action potential time resolution. The approach was sensitive enough to detect differences in latency, time-to-peak, and amplitude of capsaicin-induced ion transients along the terminal neurites. Using this approach, we found that capsaicin evokes distinctive calcium signals along the neurite. At the terminal, the signal was insensitive to voltage-gated sodium channel blockers, and showed slower kinetics and smaller signal amplitudes, compared with signals that were measured further up the neurite. These latter signals were mainly abolished by sodium channel blockers. We propose this ultrafast optical recording approach as a model for studying peripheral terminal signaling, forming a basis for studying pain mechanisms in normal and pathological states. $\odot$ The Authors. Published by SPIE under a Creative Commons Attribution 3.0 Unported License. Distribution or reproduction of this work in whole or in part requires full attribution of the original publication, including its DOI. [DOI: 10.1117/1.JBO.22.7.076010]
\end{abstract}

Keywords: nociceptive terminals; fast $\mathrm{Ca}^{2+}$ imaging; $\mathrm{Na}^{+}$imaging; pain; Campenot compartmentalized culture.

Paper 170110RRR received Feb. 16, 2017; accepted for publication Jun. 26, 2017; published online Jul. 14, 2017.

\section{Introduction}

The detection of painful stimuli takes place at the nociceptive peripheral sensory nerve terminals in the skin or the viscera. These miniature structures ( 0.5 to $5 \mu \mathrm{m}$ in diameter, Fig. 1$)$ are pivotal for the detection of noxious stimuli. ${ }^{1,2}$ Consequently, the comprehensive understanding of nociception and hence mechanisms of pathological pain can be achieved only by detailed exploration of nociceptive terminal function. However, not much is known about their properties because they are barely accessible by conventional electrophysiological methods. The saphenous skin-nerve preparation and ex vivo somatosensory system models developed by Reeh $^{3}$ and Koerber and Woodbury, ${ }^{4}$ respectively, as well as other models using extracellular recordings from nerve fibers ${ }^{5-7}$ monitor axonal activity following activation of the terminals, in different conditions, but not the activity at the terminals themselves. Electrophysiological recordings and ion imaging from terminal axons provided essential information about $\mathrm{Na}^{+}$currents, which underlie action potential generation $^{8-10}$ and terminal $\mathrm{Ca}^{2+}$ signaling. ${ }^{11,12}$ Nevertheless, spatial constraints of the electrophysiological method make it unsuitable for studying biophysical properties of signal propagation along the cylindrical terminal and distal axon. Conventional imaging assays overcome these spatial limitations; however, they are lacking in sufficient temporal resolution crucial for

*Address all correspondence to: Alexander M. Binshtok, E-mail: alexander binshtok@ekmd.huji.ac.il exploring ion dynamics underlying action potential generation and propagation. Many molecular and biophysical aspects of nociception have been described using nociceptor cell bodies, situated in dorsal root ganglions (DRG) or trigeminal ganglions. ${ }^{13-16}$ The functional environment ${ }^{17-19}$ and geometry of terminals differ from that of cell bodies, and likely, their passive membrane properties, density, and specific repertoire of transducer and voltage-gated channels. Hence, detailed characterization of ion signal onset, kinetics, and magnitude, along the terminals and terminal axons, have yet to be achieved, albeit being highly valuable for understanding nociceptive physiology. Consequently, the basic questions in nociceptive physiology, such as where action potentials are generated and how they propagate along nonmyelinated tiny axons in normal and pathological conditions, still remain obscure.

Here, we introduce a new approach for high-temporal resolution optical recordings of ion dynamics from a single nociceptive neurite terminal in vitro. This approach allows us to monitor capsaicin-induced calcium $\left(\mathrm{Ca}^{2+}\right)$ and sodium $\left(\mathrm{Na}^{+}\right)$dynamics at the terminal neurites and characterize their propagation along the distal neurites. Using this approach, we have demonstrated that the capsaicin-induced $\mathrm{Ca}^{2+}$ and $\mathrm{Na}^{+}$signals change as they propagate from the terminal and along the neurite. We showed that capsaicin-induced calcium signals initiated at the terminals were not dependent on voltage-gated $\mathrm{Na}^{+}$channels, whereas signals measured $15 \mu \mathrm{m}$ away and onward, toward the cell body, were faster, stronger, and at least partially mediated by voltage-gated $\mathrm{Na}^{+}$channels. 
(a)

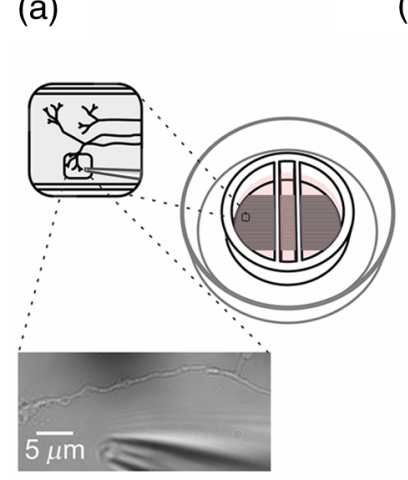

(b)
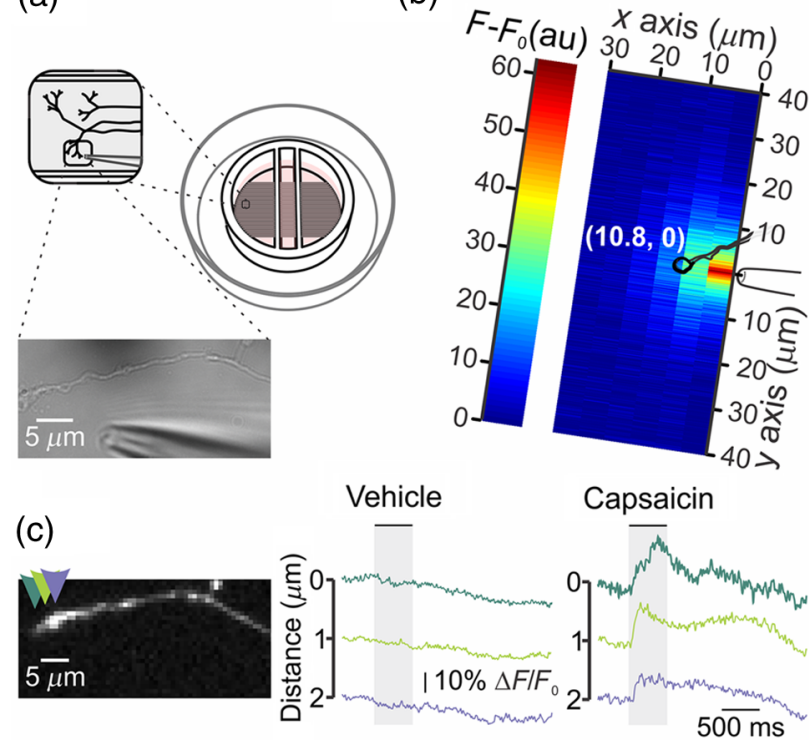

Fig. 1 GCaMP6s-based $\mathrm{Ca}^{2+}$ imaging of nociceptive-cultured DRG neurite terminals and distal processes. (a) Scheme depicting the experimental protocol (see Sec. 2). The cell bodies of DRG neurons were seeded in the middle compartment of the chamber and neurites were induced to grow to the lateral compartment. Upper inset, schematic representation of the neurite terminals and stimulating pipette. Lower inset, bright field image of a representative neurite terminal and puff pipette location. (b) Puff calibration experiment. Dispersion of a fluorophore (sulfarhodamine 101, $2 \mu \mathrm{M}$ ) following a 500-ms puff (see Sec. 2) is presented in relation to the representative neurite terminal [rendered from (a), lower inset], as a reduction profile of the fluorescence $\left(F-F_{0}\right.$, indicated by arbitrary units-au, and color coded, left) with distance from the puff pipette located at coordinates of $x, y=0$ (see Sec. 2). Coordinates of the neurite tip are indicated in parenthesis. Note that on the $x$-axis, the fluorescent intensity was reduced by about $50 \%$ when reaching a distance of $10 \mu \mathrm{m}$ from the tip of the puff pipette. On the $y$-axis, intensity diminished almost completely after $15 \mu \mathrm{m}$. (c) Left, fluorescent image of the neurite's terminal process expressing GCaMP6s. Arrows indicate locations of ROIs from which the recordings shown to the right were performed. Right, representative traces of changes in $\left[\mathrm{Ca}^{2+}\right]_{\text {in }}$ following focal puff application of vehicle and 300-nM capsaicin (far right) measured every $1 \mu \mathrm{m}$ along the terminal (" 0 " indicates the terminal tip), at a sample rate of $1000 \mathrm{fps}$. Note that the puff of vehicle does not elicit a response. Also note the spatial resolution, allowing analysis of ROls situated $1 \times 1 \mu \mathrm{m}$ apart, along the terminal. Shadowed areas indicate time period of puff application of vehicle or capsaicin. Representative of 20 out of $20(20 / 20)$ terminals.

\section{Materials and Methods}

\subsection{Animals}

All animal procedures were approved by the Ethics Committee of the Hebrew University. Five- to six-week-old male C57BL/6 mice were used.

\subsection{Dorsal Root Ganglion Compartmental Cultures}

The compartmented chamber dishes were prepared similarly to that described by Campenot et al. ${ }^{20}$ Briefly, poly-lysine- and laminin-coated dishes were scraped with a pin-rake $(200-\mu \mathrm{m}$ width of pins; Tyler Research) to create a series of parallel tracks on the laminin coating, limiting neurite growth to the areas left with laminin, in parallel lanes. A droplet of $0.4 \%$ methylcellulose
(Sigma) in DMEM (Biological Industries) was spread to cover the scratched region where the Teflon compartmentalized chamber is placed. Teflon compartmentalized chambers (Teflon dividers, Camp10 or Camp320, Tyler Research) were attached to the culture dish with silicon grease (Dow Corning), applied with a syringe grease applicator (CAMP-GLSS, Tyler Research). The central compartment was used for plating the cell soma, flanked by peripheral compartments, containing lanes in which neurites can extend [Fig. 1(a)].

DRG culture preparation: Adult mice were deeply anesthetized (3\% isoflurane), and DRG were isolated and dissociated in a manner similar to that described previously ${ }_{15}^{15}$ with the following modifications: dissected ganglia were placed in ice-cold DMEM (Biological Industries), pelleted, and reconstituted for enzymatic digestion in solution containing $5 \mathrm{mg} / \mathrm{ml}$ collagenase and $1 \mathrm{mg} / \mathrm{ml}$ dispase II (Roche) for $45 \mathrm{~min}$. Cells were triturated in the presence of $50 \mathrm{U}$ DNase I (Sigma) and centrifuged through 10\% bovine serum albumin (Sigma). The cell pellet was resuspended in $1 \mathrm{ml}$ of Neurobasal (Gibco) medium containing B27 supplement (Invitrogen), penicillin and streptomycin (Biological Industries), $100 \mathrm{ng} / \mathrm{ml}$ 2.5S NGF (Promega), and $2 \mathrm{ng} / \mathrm{ml} \mathrm{GDNF}$ (Sigma). Cells were plated on to polylysine- $(500 \mu \mathrm{g} / \mathrm{ml})$ and laminin- $(5 \mathrm{mg} / \mathrm{ml})$ coated $35-\mathrm{mm}$ glass bottom tissue culture dishes [World Precision Instruments (WPI)] in the middle compartment of the compartmented chambers [Fig. 1(a), see also Ref. 20].

On the day of seeding the cells (day 0 ), both chambers contained the above-mentioned Neurobasal media with $100 \mathrm{ng} / \mathrm{ml}$ 2.5S NGF; thereafter, the concentrations of NGF were altered to promote the sprouting neurites to grow toward the distal chambers as follows (cell bodies and peripheral compartments, respectively): day $1: 10$ and $100 \mathrm{ng} / \mathrm{ml}$; day $2: 1$ and $10 \mathrm{ng} / \mathrm{ml}$; and days 5, 8, and day 11:0 and $1 \mathrm{ng} / \mathrm{ml}$.

Cultures were maintained at $37^{\circ} \mathrm{C}$ in a humidified incubator containing 5\% $\mathrm{CO}_{2}$. The cultured DRG cell somata underwent viral coinfection on either of 8- to 10-days postseeding. Imaging experiments of neurite terminals were carried out on days 11 to 14 from seeding. Teflon dividers were removed prior to the experiment.

\subsection{Viral Vectors}

To measure the changes in intraterminal and intraneuritic $\mathrm{Ca}^{2+}$ concentrations $\left(\left[\mathrm{Ca}^{2+}\right]_{\text {in }}\right)$, the adeno-associated virus serotypes 1 or 6 , carrying an expression cassette for the genetically encoded $\mathrm{Ca}^{2+}$ indicator (GCaMP6s) or the mRuby florescent protein indicator (under a CMV promoter; ELSC Virus Core Facility), were used. Cells were infected by the two viruses simultaneously.

\subsection{Imaging}

Image data were processed by Neuroplex software (RedShirt Imaging) and analyzed using pCLAMP 10.2 (Molecular Devices) and OriginPro 9.0.0 (OriginLab Corporation).

Number of repeats in this study ( $n$ 's) refers to the number of terminals examined in each experiment. In the $\mathrm{Ca}^{2+}$ imaging assay, each analyzed terminal was from a culture made from a different animal. For the $\mathrm{Na}^{+}$imaging assay, $n$ reflects different terminals in different dishes, however, not all from different animals.

All analyses were performed on the processes in the same field of view. 
We used a short imaging protocol of up to $5 \mathrm{~s}$ to avoid photo damage, which occurred during longer exposure, probably due to the recurrent excitation with high-intensity ultraviolet and blue excitation wavelengths used here.

\subsection{1 $\mathrm{Ca}^{2+}$ imaging}

Fluorescent excitation was performed with a $75 \mathrm{~W}$ Xenon arc lamp (Lambda DG4; Shutter Instruments) and a GFP filter set (Ex 480, Em 535, dichroic Lp 510; Chroma). An inverted microscope (Eclipse Ti; Nikon) equipped with a $\times 60$ oil 1.4NA objective, Epi-Fl attachment, and perfect focus system was used. For bright field imaging and fluorescent still image acquisition, an Exi Aqua monochromator camera (QImaging) and Nikon elements AR software (Nikon) were used. Changes in intraterminal and intraneuritic fluorescence were acquired using a backilluminated $80 \times 80$ pixel cooled CCD camera (NeuroCCDSMQ; RedShirt Imaging), controlled by Neuroplex software (RedShirtImaging). In these conditions, pixel size was $1 \times$ $1 \mu \mathrm{m}$. This spatial resolution was sufficient for analyzing the signal propagation along terminals and distal neurites, even though in some locations the diameter of the structure $(0.5$ to $5 \mu \mathrm{m}$ ) could have been less than the camera resolution.

Images were acquired at 1000 frames/second (fps).

\subsection{2 $\mathrm{Na}^{+}$imaging}

To measure changes in intraterminal and intraneuritic $\mathrm{Na}^{+}$concentrations $\left(\left[\mathrm{Na}^{+}\right]_{\text {in }}\right)$ in vitro, the cell-permeable $\mathrm{Na}^{+}$indicator SBFI-AM (Molecular Probes) was used. Five-millimolar stock was prepared in dimethyl sulfoxide with $10 \%$ Pluronic F-127. Cells were incubated for $1 \mathrm{~h}$ with a $5 \mu \mathrm{M}$ final concentration of SBFI-AM in the culture media, followed by a 30-min rinse period. Images were acquired at 125 fps. Florescent excitation was performed using a CoolLed fluorescence excitation system, using a 380-nm LED and a Fura2 filter set (Ex: 380 nm, Em: $510 \mathrm{~nm}$, dichroic Lp: $400 \mathrm{~nm}$; Chroma). To compensate for time-dependent decrease in fluorescence, due to fluorophore bleaching, fitted linear curves derived from the first $500 \mathrm{~ms}$ of the acquired data were subtracted from the recorded data.

\subsection{Solutions}

All imaging experiments were performed in standard external solution (SES) composed of $145 \mathrm{mM} \mathrm{NaCl}, 5 \mathrm{mM} \mathrm{KCl}, 2 \mathrm{mM}$ $\mathrm{CaCl}_{2}, 1 \mathrm{mM} \mathrm{MgCl}$, $10 \mathrm{mM}$ glucose, and $10 \mathrm{mM}$ HEPES ( $\mathrm{pH}=7.4)$.

Capsaicin $(300 \mathrm{nM})$ or vehicle (SES with $0.3 \mu \mathrm{l} / \mathrm{ml}$ ethanol) was focally puff applied (500 ms puff, 2 psi, PV820 Pneumatic PicoPump; WPI) by a pipette with 4 to $6 \mathrm{M} \Omega$ resistance, when filled with SES. The application regime was commanded by a Digidata 1440 A/D interface (Molecular Devices).

In some experiments, the $\mathrm{Na}^{+}$channel blocker, tetrodotoxin (TTX, $10 \mu \mathrm{M}$; Alomone Labs) together with a Na(v) 1.8 channel blocker, A803467 (10 $\mu M$; Tocris), were applied to bath for $10 \mathrm{~min}$ before the recordings.

\subsection{Puff Calibration}

We calibrated the dispersion profile of puffed substances by measuring changes in the sulfarhodamine $(2 \mu \mathrm{M})$ fluorescent intensity profile with distance from the pipette tip [Fig. 1(b)] according to
$\frac{F_{x 1, y 1}}{F_{x 0, y 0}} \times C_{x 0, y 0}=C_{x 1, y 1}$,

where $F$ is the fluorescence intensity at $x, y$ coordinates and $C$ is the concentration.

\subsection{Data Analysis}

To measure changes in $\left[\mathrm{Ca}^{2+}\right]_{\text {in }}$ within a terminal at the highest possible resolution our conditions permit, the data were collected from $1 \times 1 \mu \mathrm{m}$ sized regions of interest (ROIs) [1 pixel; Fig. 1(c)]. To examine changes in $\mathrm{Ca}^{2+}$ concentration propagating along the neurite and to improve the signal-to-noise ratio, the data were collected using Kernel smoother from $2 \times 2 \mu \mathrm{m}$ sized ROIs ( $2 \times 2$ pixels; Fig. 2$)$, which were distributed every $5 \mu \mathrm{m}$ along the neurite's terminal and processes, dividing the terminals into $m$ segments. All the data were analyzed from the ROIs delimiting the examined segments, and the size of the ROI was neglected when the actual distance was calculated. Therefore, the distance is represented as an estimated location rather than an absolute distance.

The time of onset of the signal was measured as the time at the onset of a clear sharp deviation from fluorescent base level measured locally, while analyzing the trace from its positive peak toward time " 0 ." All the values were subtracted from the value at the terminal tip $(x=0)$ of the corresponding terminal.

Difference in time of onset between two adjacent segments, i.e., the delay in onset at one segment compared with the time of onset at the previous segment, were calculated according to

$t_{m}-t_{m-1}$,

where $m$ is the $m$ 'th segment.

The fluorescence propagation velocity within $5 \mu \mathrm{m}$ segments was calculated according to

$v=\frac{5}{t_{m}-t_{m-1}}$,

where $v$ is the fluorescence propagation velocity and 5 is the distance between each ROI in micrometers $(\mu \mathrm{m})$.

Maximum rate of rise of $\mathrm{Ca}^{2+}$ transients was calculated as $\max (\mathrm{d} F / \mathrm{d} t)$ at different locations along the neurite terminal. The values were normalized to the value of the terminal tip of the corresponding terminal and plotted as a function of distance from the terminal tip.

All averaged data are presented as the mean \pm SEM. Assessment of statistical significance of differences between means was performed with repeated-measures of analysis of variance (ANOVA), with posthoc Bonferroni, as found appropriate.

\section{Results and Discussion}

To study processing of nociceptive information directly at its most relevant location, with sufficient time and space resolution, we introduced an approach for ultrafast optical recording of cultured nociceptive processes. To that end, we used compartmented chambers [Fig. 1(a), see Sec. 2] that have been previously used to study neurites of DRG neurons. ${ }^{21-24}$ In these chambers, DRG cell bodies are prompt to grow neurites, and terminal processes can be easily identified and accessed [Figs. 1(a) and 1(c)].

We selectively stimulated these neurite terminals with a calibrated focal puff application [Fig. 1(b)] of capsaicin, an agonist of the noxious heat-sensitive transient receptor potential cation 
(a)

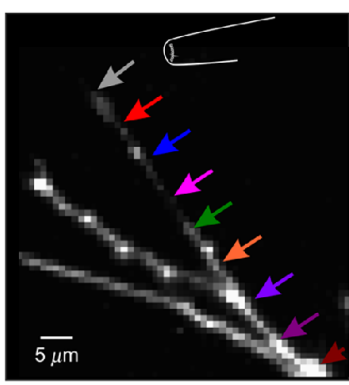

(d)

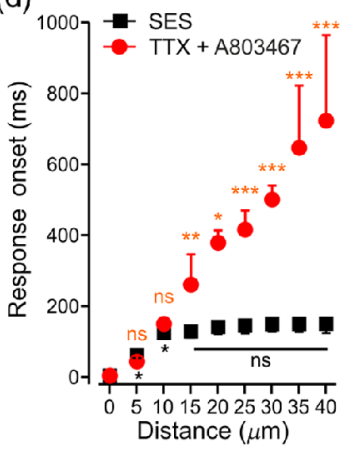

(b)

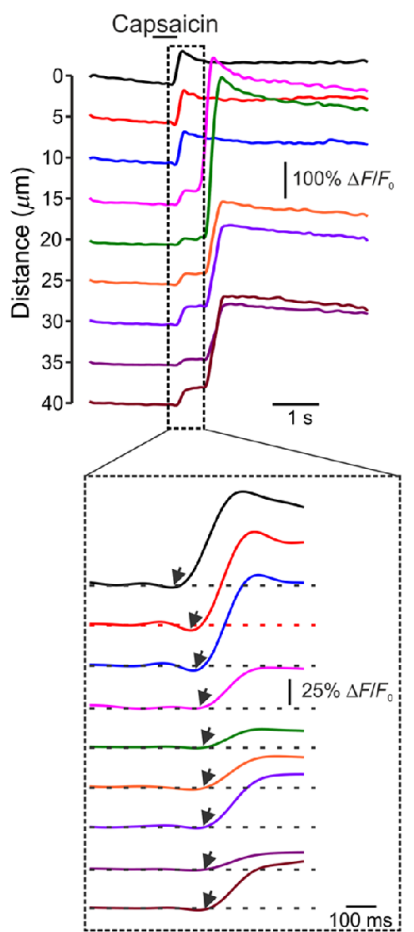

(e)

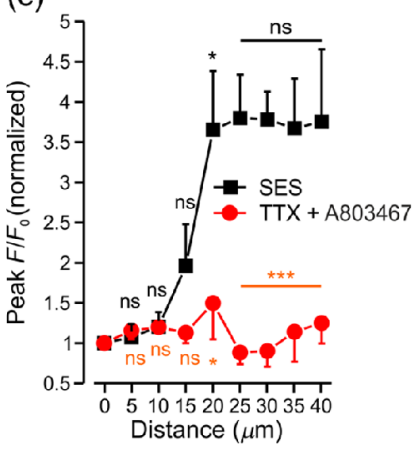

(c) TTX + A803467

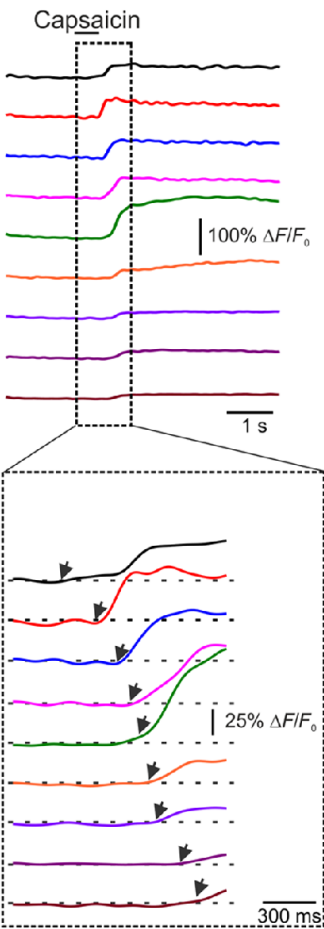

(f)

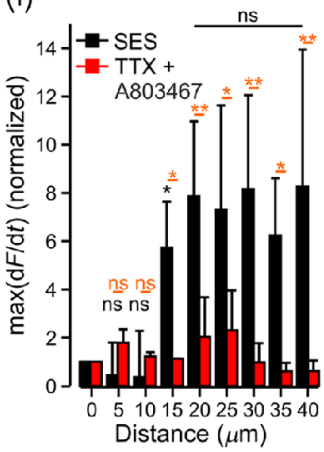

Fig. 2 Propagation of capsaicin-induced $\mathrm{Ca}^{2+}$ transients along the neurite terminal. (a) Fluorescence image of representative nociceptive terminals expressing GCaMP6s with illustration of pipette location and with the arrows indicating the locations of ROls from which the measurements shown in (b) and (c) were performed. (b, c) Representative traces of capsaicin-induced changes in $\left[\mathrm{Ca}^{2+}\right]_{\text {in }}$ measured every $5 \mu \mathrm{m}$ at distances shown in left, from the terminal tip before (b) and $10 \mathrm{~min}$ after (c) application of $10-\mu \mathrm{M}$ TTX together with $10-\mu \mathrm{M}$ A803467. Changes in fluorescents were measured at $1000 \mathrm{fps}$. Note the substantial increase in signal amplitude after $15 \mu \mathrm{m}$, which was abolished by applying TTX and A803467. Insets: Expanded timescale of the traces presented in (b) and (c) emphasizing the signal onset. Arrows indicate calculated time of signal onset (see Sec. 2). Note that the timescale of the inset for (b) is further expanded to better illustrate the differences in time of signal onset along the neurite in the control conditions. (d) Time of onset of capsaicin-induced $\mathrm{Ca}^{2+}$ signals measured from terminal tip (point "0") in control conditions (SES, black squares) and after bath application of TTX together with A803467 (red circles), plotted versus distance from the terminal tip. Note much smaller changes in time of onset after $15 \mu \mathrm{m}$ in control conditions. Application of TTX together with A803467 significantly increased the difference in time of onset after $15 \mu \mathrm{m}$. ns, not significant; ${ }^{*} p<0.05,{ }^{\star *} p<0.01$, ${ }^{\star \star \star} p<0.001$; black letters and symbols, comparison between values measured at each location in control condition, $n=4$, one-way ANOVA with posthoc Bonferroni; orange letters and symbols, comparison between "SES" and "TTX + A803467;" $n=2$; two-way ANOVA, with posthoc Bonferroni. (e) Peak amplitudes of capsaicin-induced changes in fluorescence, measured every $5 \mu \mathrm{m}$ and plotted as a function of distance from terminal tip, in control conditions (SES, black) and after (red) application of TTX together with A803467. Note that in control conditions, signal amplitude significantly increased after $15 \mu \mathrm{m}$. TTX together with A803467 did not affect the signal measured at the first $15 \mu \mathrm{m}$, but significantly reduced the signal amplitude thereafter. Symbols and statistics as in (d). (f) Maximum rate of rise of $\mathrm{Ca}^{2+}$ transients, $\max (\mathrm{d} F / \mathrm{d} t)$, at the different locations along the neurite terminal in control condition (SES, black), and TTX together with A803467 (red) plotted as a function of distance from the terminal tip. Note that in control conditions, the rise rate significantly increased at $\sim 15 \mu \mathrm{m}$ and plateaued afterwards. Application of TTX together with A803467 prevented the increase in rise rate. Statistics as in (d). 
channel subfamily V member 1 (TRPV1) channel, expressed by nociceptive neurons. ${ }^{25} \mathrm{We}$ placed the pipette, containing 300 $\mathrm{nM}$ capsaicin, about $10 \mu \mathrm{m}$ from the terminal tip [Figs. 1(a) and 1(b)] because at this distance, the application of vehicle did not induce changes in GCaMP6s fluorescence [Fig. 1(c); $n=20$ terminals] or detectable movement of the processes. First, we calibrated the dispersion profile of the puffed substances [see Sec. 2; Fig. 1(b)]. Our data show that the predicted concentration at the terminal tip is $\sim 50 \%$ of the initial value [Fig. 1(b)], i.e., in our conditions, the concentration of capsaicin at the terminal is close to the half maximal effective concentration $\left(\mathrm{EC}_{50}\right)$ of TRPV1 channels to capsaicin $\left(\sim 150 \mathrm{nM}^{26}\right)$. This is an estimate of the peak concentration during a 500-ms puff, which dissipates in the medium when the puff ends. Additionally, considering that the minimal concentration of capsaicin to activate TRPV1 channels is $\sim 30 \mathrm{nM},{ }^{26}$ the prominent decrease in concentration along the $y$-axis [Fig. 1(b)] renders the concentration mostly ineffective once it reaches other parts of the fiber or adjacent terminals. Indeed, in all the performed recordings, we did not observe $\mathrm{Ca}^{2+}$ responses in neighboring terminal branches of the neurite.

We measured capsaicin-induced increase in $\left[\mathrm{Ca}^{2+}\right]_{\text {in }}$ at the neurite's terminal process using the genetically encoded $\mathrm{Ca}^{2+}$ indicator GCaMP6slow [GCaMP6s, Fig. 1(c)]. We chose the slow variant of the GCaMP6 indicator due to its better signalto-noise ratio and sensitivity (compared with other GCaMP6 variants ${ }^{27}$ ), which enabled the detection of weak fluorescent emission signals from fine neurite processes. To study transducer channel- and action potential-mediated changes in terminal $\left[\mathrm{Ca}^{2+}\right]_{\text {in }}$, we optically recorded changes in terminal GCaMP6s fluorescent intensity at a sampling rate of $1000 \mathrm{fps}$. As it has been shown that the half width of the action potential recorded from the distal neurite of nociceptive neurons is $\sim 2.5 \mathrm{~ms},{ }^{8}$ this sampling rate should be sufficient to detect $\mathrm{Ca}^{2+}$ reflections of single action potentials. We achieved this using a $60 \times$ oil 1.4NA Plan Apo objective, in combination with a fast acquisition back-illuminated $80 \times 80$ pixel CCD camera (see Sec. 2). Puff application of capsaicin, but not vehicle [Fig. 1(c)], led to prominent increase in GCaMP6s fluorescence, detectable at a spatial resolution of $1 \times 1 \mu \mathrm{m}$ along the terminal tip [Fig. 1(c)].

Next, utilizing the high functional spatial resolution of our approach, which allows precise tracing of signal propagation along the terminal, we measured the amplitude, time of onset, and rise rate of capsaicin-induced $\mathrm{Ca}^{2+}$ signals along the terminal and terminal neurite [Figs. 2(b), 2(d)-2(f), black]. It is noteworthy that due to short acquisition time (see Sec. 2), we could not explore the mechanisms underlying the decay phase of the capsaicin-induced response at the neurite's processes and, therefore, we focused our efforts on the rising phase of the response.

We analyzed the difference in time of onset between two adjacent segments, consecutively for all adjacent segments (see Sec. 2), which reflects the time it took the signal to travel along a segment. We found that the signal traveled along $m_{1}$ for $58.5 \pm 10 \mathrm{~ms}, m_{2}$ for $62.2 \pm 10 \mathrm{~ms}$, and $m_{3}$ for $15.6 \pm 5 \mathrm{~ms}$ $(n=4)$. After segment $m_{3}$, time of onset difference between two ROIs was $3.2 \pm 2 \mathrm{~ms}$ [segments $m_{3}$ to $m_{8}$; Fig. 2(d), black, $n=4]$. Using these data, we calculated the fluorescent propagation velocity of capsaicin-induced $\mathrm{Ca}^{2+}$ signals between segments (see Sec. 2) and demonstrated that the signal propagated along $m_{1}$ with a velocity of $97.2 \pm 23 \mathrm{~nm} / \mathrm{ms}, m_{2}$ with
$85.2 \pm 11 \mathrm{~nm} / \mathrm{ms}$, and $m_{3}$ with $382 \pm 95 \mathrm{~nm} / \mathrm{ms} \quad(n=4)$. Signals propagated between segments $m_{1}$ to $m_{3}$ did not vary in their response peak amplitude or maximum rise rate $[\max (\mathrm{d} F / \mathrm{d} t)$; Figs. 2(b), 2(e), 2(f) black]. After $\sim 15 \mu \mathrm{m}$ (segment $\left.m_{3}\right)$, the fluorescence propagation velocity increased significantly and reached a peak of $870 \pm 300 \mathrm{~nm} / \mathrm{ms}$ at $m_{4}$ $(\sim 20 \mu \mathrm{m})$, and the speed of propagation between $5 \mu \mathrm{m}$ segments remained similar along the rest of the measured terminal ( $p=0.47$, one-way ANOVA, $n=4)$. It is noteworthy that the values for propagation velocity that we have measured represent the propagation of fluorescent signals along the process and not the electrical conductance. The propagation of GCaMP6s fluorescence is dependent on electrical conductance; however, there are many additional factors that are involved, such as the $\mathrm{Ca}^{2+}$ channels' and indicator kinetics (see also Ref. 28).

Remarkably, at $15 \mu \mathrm{m}$ (segment $m_{3}$ ), a two-phase response appeared [Fig. 2(b)]. While the first phase was similar to the one observed at the terminal tip ( $p=0.35$ for rise rate; $p=0.58$ for the amplitude, one-way ANOVA with posthoc Bonferroni), the second phase possessed a significantly larger peak amplitude and maximum rise rate $[p<0.05$, one-way ANOVA, $n=6$ terminals; Figs. 2(e), 2(f) black], reaching a plateau of about 3.5 times larger response peak amplitude and 7 times larger rise rate at the maximum. This large and fast component was completely abolished following application of a combination of TTX and A803467 [Figs. 2(c), 2(d)-2(f) red] to block TTX-s- and Na (v)1.8-mediated TTX-r Na ${ }^{+}$channels, ${ }^{29}$ which are expressed by nociceptive terminals. ${ }^{8}$ Moreover, blocking $\mathrm{Na}(\mathrm{v})$ channels delayed the time of onset [Fig. 2(d), red], reduced the fluorescence propagation velocity to $57 \pm 11 \mathrm{~nm} / \mathrm{ms}$, and decreased the peak amplitude and maximal rise rate [Figs. 2(e) and 2(f), red] of capsaicin-evoked responses recorded along the neurite after $\sim 15 \mu \mathrm{m}$, such that the values become similar to those recorded at the first $15 \mu \mathrm{m}$ [Figs. 2(e) and 2(f)]. These data suggest that the increase in $\left[\mathrm{Ca}^{2+}\right]_{\text {in }}$ beyond the $\sim 15 \mu \mathrm{m}$ is partially dependent on activation of voltage-gated $\mathrm{Na}^{+}$channels.

We further supported the latter notion by directly measuring capsaicin-induced $\mathrm{Na}^{+}$fluxes in the neurite's terminal process using the $\mathrm{Na}^{+}$indicator SBFI-AM. Imaging of $\mathrm{Na}^{+}$fluxes at different locations along the terminal revealed that application of capsaicin induced a small transient increase in $\left[\mathrm{Na}^{+}\right]_{\text {in }}$, in the neurite's terminal tip and a larger step-like $\mathrm{Na}^{+}$signals at a distance from the tip [Fig. 3(b), $n=3$ ].

It is noteworthy to mention that TRPV1 channels are also permeable to $\mathrm{Na}^{+} .{ }^{30}$ Thus, the signal at the terminal tip may reflect $\mathrm{Na}^{+}$influx via active TRPV1 channels. The fast and step-like nature of the signals recorded away from the neurite may underlie burst-like firing of the action potentials. ${ }^{31}$ From a methodological point of view, this in vitro approach, which is based on a distinctive compartment containing only neurite terminals, is currently the only approach allowing direct study of terminal $\mathrm{Na}^{+}$dynamics. It is advantageous over an in vivo assay as the usage of AM-based dyes in vivo will likely produce a strong background noise due to AM dye loading of not only the terminals but also nonneuronal cells in the imaged area. The lack of genetically encoded $\mathrm{Na}^{+}$indicators for specific measurements of $\mathrm{Na}^{+}$flux in distinct cell types render measurements from small structures in vivo or ex vitro extremely challenging. Importantly, low initial fluorescence intensity of SBFI-AM in the neurite's terminal, due to multiple factors (e.g., relatively low passage of SBFI excitation wave length by the objective and intraterminal loading) allowed us to sample 
(a)

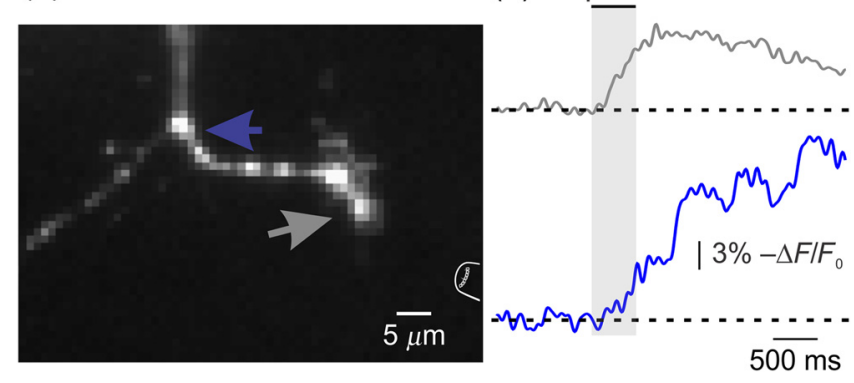

Fig. 3 Capsaicin-induced $\mathrm{Na}^{+}$transients vary along the neurite terminal. (a) Representative image of a nociceptive terminal process loaded with SBFI-AM. Note at the lower right corner, the illustration of pipette location. Arrows indicating locations of ROls from which the recordings shown in (b) were performed. (b) Representative traces of changes in $\left[\mathrm{Na}^{+}\right]_{\text {in }}$ measured at the terminal tip (gray) and $20 \mu \mathrm{m}$ from the terminal tip (blue), with a sampling rate of $125 \mathrm{fps}$. Note transient increase in $\left[\mathrm{Na}^{+}\right]_{\text {in }}$ at the tip and step-like continuous response at $20 \mu \mathrm{m}$. Dotted lines indicate baseline fluorescence. Traces indicating decrease in florescence emission after excitation with $380 \mathrm{~nm}$. Shadowed area indicates time period of puff application of capsaicin.

capsaicin-induced intraterminal changes of $\mathrm{Na}^{+}$, only at 125 fps.

Altogether our data suggest that the nociceptive neurite's terminals and processes, in terms of $\mathrm{Ca}^{2+}$ dynamics and its dependence on $\mathrm{Na}^{+}$channel blockers, are composed of two functionally separated zones. Our results imply that signal generation and propagation in the first $15 \mu \mathrm{m}$ is not mediated by voltage-gated $\mathrm{Na}^{+}$channel-induced depolarization. This $\mathrm{Ca}^{2+}$ signaling could be mediated by $\mathrm{Ca}^{2+}$-permeable TRPV1 channels, low threshold voltage-gated $\mathrm{Ca}^{2+}$ channels, $\mathrm{Ca}^{2+}$. induced $\mathrm{Ca}^{2+}$ release, and capsaicin-mediated $\mathrm{Ca}^{2+}$ release from TRPV1-expressing internal stores. ${ }^{32}$ Given the focality of our application and the susceptibility of the late response to $\mathrm{Na}^{+}$ channel blockers, TRPV1-mediated $\mathrm{Ca}^{2+}$ release from internal stores has a lesser contribution to the capsaicin-induced $\mathrm{Ca}^{2+}$ response, which we observed after $15 \mu \mathrm{m}$. Hence, as suggested by our data, signal propagation beyond the first $15 \mu \mathrm{m}$ is largely driven by voltage-gated $\mathrm{Na}^{+}$channels leading to $\mathrm{Ca}^{2+}$ elevation probably via voltage-gated $\mathrm{Ca}^{2+}$ channels and $\mathrm{Ca}^{2+}$-induced $\mathrm{Ca}^{2+}$ release. Interestingly, the maximal propagation velocity, we have measured from terminal neurites of nociceptive processes, was substantially smaller than those measured using single fiber recordings from mice sciatic-tibial nerve in vivo. ${ }^{6}$ This discrepancy could result from the differences between electrical conductance and fluorescent propagation, as stated above. Indeed, the velocities of fluorescence propagation that we and others ${ }^{28}$ have measured in cultured neurites are slower than that measured for propagation of electrical signals. ${ }^{6}$

In this study, we have analyzed the activity of individual terminal neurites, which are easily identified and traced. It is difficult, however, to trace and record individual neurite activity along its full length because proximal neurites in culture create dense bundles of neurites. ${ }^{20,24}$ Utilization of our approach on individually labeled neurons, via micropipette injection of a spectrally different dye, may overcome this difficulty and provide essential information about the calcium dynamics along different compartments of peripheral processes.

In conclusion, we have introduced an approach for studying nociceptive terminal processes by bringing together and utilizing fast and sensitive optical recordings of ion dynamics from easily accessible cultured neurite terminals. Altogether, the data shown here demonstrate that our methodology gains in sensitivity and temporal acquisition capabilities, compared with standard imaging techniques, allowing fast optical recordings of weak signals from minute nociceptive terminal processes. Moreover, our approach gains in functional spatial resolution, compared with single electrode electrophysiological approaches, permitting characterization of signal propagations along the terminal and terminal neurites by sampling the signals in action potential relevant time resolution. Thus, the ultrafast and high-resolution optical recording technique described here provides the only tool for a detailed study of a nociceptive terminal functional molecular network, which underlies noxious stimuli detection and transmission in normal and pathological conditions. This could advance the very much needed knowledge for understanding pain physiology and pathophysiology. Moreover, this platform can be utilized to enable studying the terminals of other neuronal types, such as other primary sensory neuron terminals.

\section{Disclosures}

No conflicts of interest, financial or otherwise, are declared by the authors.

\section{Acknowledgments}

Support is gratefully acknowledged from the Deutsch-Israelische Projectkooperation program of the Deutsche Forschungsgemeinschaft (DIP) grant agreement BI 1665/1-1ZI1172/12-1 (RHG, BK, SL, and AMB); European Research Council under the European Union's Seventh Framework Programme (FP7/ 2007-2013)/ERC grant agreement no. 260914 (RHG, BK, SL, and AMB); the Jacob and Lena Joels Chair for Excellence in the Life and Medical Sciences (AMB); and the Hoffman Leadership program (RHG). We would like to thank Dr. Maya Groysman, the manager of ELSC viral core facility, for designing and providing viruses used in this study.

\section{References}

1. T. Hucho and J. D. Levine, "Signaling pathways in sensitization: toward a nociceptor cell biology," Neuron 55(3), 365-376 (2007).

2. A. M. Binshtok, "Mechanisms of nociceptive transduction and transmission: a machinery for pain sensation and tools for selective analgesia," Int. Rev. Neurobiol. 97, 143-177 (2011).

3. P. W. Reeh, "Sensory receptors in mammalian skin in an in vitro preparation," Neurosci. Lett. 66(2), 141-146 (1986).

4. H. R. Koerber and C. J. Woodbury, "Comprehensive phenotyping of sensory neurons using an ex vivo somatosensory system," Physiol. Behav. 77, 589-594 (2002).

5. M. Tal and E. Eliav, "Abnormal discharge originates at the site of nerve injury in experimental constriction neuropathy (CCI) in the rat," Pain 64(3), 511-518 (1996).

6. D. M. Cain, S. G. Khasabov, and D. A. Simone, "Response properties of mechanoreceptors and nociceptors in mouse glabrous skin: an in vivo study," J. Neurophysiol. 85(4), 1561-1574 (2001).

7. P. R. Burgess and E. R. Perl, "Myelinated afferent fibres responding specifically to noxious stimulation of the skin," J. Physiol. 190(3), 541-562 (1967).

8. D. V. Vasylyev and S. G. Waxman, "Membrane properties and electrogenesis in the distal axons of small dorsal root ganglion neurons in vitro," J. Neurophysiol. 108(3), 729-740 (2012).

9. J. A. Brock, E. M. McLachlan, and C. Belmonte, "Tetrodotoxin-resistant impulses in single nociceptor nerve terminals in guinea-pig cornea," J. Physiol. 512(Pt. 1), 211-217 (1998). 
10. R. W. Carr et al., "Action potential initiation in the peripheral terminals of cold-sensitive neurones innervating the guinea-pig cornea," J. Physiol. 587(Pt. 6), 1249-1264 (2009).

11. T. D. Gover, J. P. Kao, and D. Weinreich, "Calcium signaling in single peripheral sensory nerve terminals," J. Neurosci. 23(12), 4793-4797 (2003).

12. L. D. Baillie et al., "Functional imaging within individual pain fibres ex vivo with optical microscopy," J. Neurosci. Methods 198(2), 274-279 (2011).

13. S. D. Dib-Hajj et al., "NaN, a novel voltage-gated Na channel, is expressed preferentially in peripheral sensory neurons and down-regulated after axotomy," Proc. Natl. Acad. Sci. U. S. A. 95(15), 8963-8968 (1998).

14. T. R. Cummins et al., "Voltage-clamp and current-clamp recordings from mammalian DRG neurons," Nat. Protoc. 4, 1103-1112 (2009).

15. S. Gudes et al., "The role of slow and persistent TTX-resistant sodium currents in acute tumor necrosis factor-alpha-mediated increase in nociceptors excitability," J. Neurophysiol. 113(2), 601-619 (2015).

16. A. M. Binshtok et al., "Nociceptors are interleukin-1beta sensors," J. Neurosci. 28(52), 14062-14073 (2008).

17. M. Ritter-Jones, S. Najjar, and K. M. Albers, "Keratinocytes as modulators of sensory afferent firing," Pain 157(4), 786-787 (2016).

18. K. M. Baumbauer et al., "Keratinocytes can modulate and directly initiate nociceptive responses," Elife 4, e09674 (2015).

19. B. Feng et al., "Experimental and computational evidence for an essential role of NaV1.6 in spike initiation at stretch-sensitive colorectal afferent endings," J. Neurophysiol. 113(7), 2618-2634 (2015).

20. R. B. Campenot, K. Lund, and S. A. Mok, "Production of compartmented cultures of rat sympathetic neurons," Nat. Protoc. 4(12), 1869-1887 (2009).

21. R. B. Campenot, "NGF uptake and retrograde signaling mechanisms in sympathetic neurons in compartmented cultures," in Results and Problems in Cell Differentiation, Vol. 48, pp. 141-158, Springer, Berlin, Heidelberg (2009).

22. O. Natera-Naranjo et al., "Identification and quantitative analyses of microRNAs located in the distal axons of sympathetic neurons," RNA 16(8), 1516-1529 (2010).

23. S. J. Fenstermacher, M. F. Pazyra-Murphy, and R. A. Segal, "Campenot cultures and microfluidics provide complementary platforms for spatial study of dorsal root ganglia neurons," in Microfluidic and Compartmentalized Platforms for Neurobiological Research E. Biffi, Ed., Vol. 103, pp. 105-124, Springer Protocols, Springer, New York (2016).

24. M. Estacion et al., " $\mathrm{Ca}^{2+}$ toxicity due to reverse $\mathrm{Na}^{+} / \mathrm{Ca}^{2+}$ exchange contributes to degeneration of neurites of DRG neurons induced by a neuropathy-associated Nav1.7 mutation,” J. Neurophysiol. 114(3), 1554-1564 (2015).

25. M. J. Caterina et al., "The capsaicin receptor: a heat-activated ion channel in the pain pathway," Nature 389(6653), 816-824 (1997).

26. F. Yang et al., "Structural mechanism underlying capsaicin binding and activation of the TRPV1 ion channel," Nat. Chem. Biol. 11(7), 518-524 (2015).
27. T. W. Chen et al., "Ultrasensitive fluorescent proteins for imaging neuronal activity," Nature 499(7458), 295-300 (2013).

28. A. Pristera, M. D. Baker, and K. Okuse, "Association between tetrodotoxin resistant channels and lipid rafts regulates sensory neuron excitability," PLoS One 7(8), e40079 (2012).

29. M. F. Jarvis et al., "A-803467, a potent and selective Nav1.8 sodium channel blocker, attenuates neuropathic and inflammatory pain in the rat," Proc. Natl. Acad. Sci. U. S. A. 104(20), 8520-8525 (2007).

30. J. N. Wood et al., "Capsaicin-induced ion fluxes in dorsal root ganglion cells in culture," J. Neurosci. 8(9), 3208-3220 (1988).

31. I. A. Fleidervish et al., " $\mathrm{Na}^{+}$imaging reveals little difference in action potential-evoked $\mathrm{Na}+$ influx between axon and soma," Nat. Neurosci. 13(7), 852-860 (2010).

32. L. J. Karai et al., "Vanilloid receptor 1 regulates multiple calcium compartments and contributes to $\mathrm{Ca}^{2+}$-induced $\mathrm{Ca}^{2+}$ release in sensory neurons," J. Biol. Chem. 279(16), 16377-16387 (2004).

Robert H. Goldstein received his BMedSc degree, magna cum laude, from the Hebrew University, Faculty of Medicine. He is currently a $\mathrm{PhD}$ candidate in neurobiology, under the supervision of Dr. Alexander Binshtok. The majority of his PhD work is focused on studying ion and voltage dynamics in peripheral sensory free nerve endings, in vivo and in vitro, both in normal and pathological states.

Ben Katz received his BSc degree in mathematics and philosophy. He moved to biological research and received his $\mathrm{PhD}$ in medical neurobiology entitled: "Structural and molecular dynamics in Drosophila photoreceptors," under the supervision of Professor Baruch Minke. He continued to a postdoc training in medical neurobiology under the supervision of Dr. Alexander Binshtok, all of which were carried at the Hebrew University. His current research is studying a unique mutation in human TRPV1 channel.

Shaya Lev is currently working as a research associate and lab manager at Hebrew University. He received his PhD in physiology from the Hebrew University, Jerusalem, Israel, in 2010. He has published around 15 papers in different journals and conference proceedings. His research areas include neuro-cellular imaging, and cellular and molecular mechanisms of TRP channels, pain and nociception.

Alexander M. Binshtok is a $\mathrm{PI}$ at the Hebrew University Medical School studying cellular and molecular mechanisms of normal and pathological pain. He received his BSc degree in physical therapy from Ben Gurion University in 1995. His graduate work, with Dr. Michael Gutnick at the Hebrew University focused on cellular and molecular characteristics of cortical neurons. He did his postdoctoral fellowship with Dr. Clifford Woolf at Harvard Medical School, where he focused on developing approaches for pain-selective anesthesia. 